\title{
Frontières
}

\section{Bibliographie indicative sur le cadavre}

\section{Patrick Bergeron}

Volume 23, numéro 1, automne 2010

URI : https://id.erudit.org/iderudit/1004032ar

DOI : https://doi.org/10.7202/1004032ar

Aller au sommaire du numéro

Éditeur(s)

Université du Québec à Montréal

ISSN

1916-0976 (numérique)

Découvrir la revue

Citer ce document

Bergeron, P. (2010). Bibliographie indicative sur le cadavre. Frontières, 23(1),

92-92. https://doi.org/10.7202/1004032ar

Ce document est protégé par la loi sur le droit d'auteur. L'utilisation des services d'Érudit (y compris la reproduction) est assujettie à sa politique d'utilisation que vous pouvez consulter en ligne.

https://apropos.erudit.org/fr/usagers/politique-dutilisation/
Cet article est diffusé et préservé par Érudit.

Érudit est un consortium interuniversitaire sans but lucratif composé de l'Université de Montréal, l'Université Laval et l'Université du Québec à Montréal. Il a pour mission la promotion et la valorisation de la recherche. https://www.erudit.org/fr/ 


\section{BIBLIOGRAPHIE INDICATIVE SUR LE CADAVRE}

Patrick Bergeron

AKASS, K. (2005). Reading Six Feet Under. TV to Die For, Londres, I.B. Tauris.

BACQUÉ, M.-F. et C. BERSAY (dir.) (2010). « Les morts dans la ville», Études sur la mort, n 137 (novembre).

BARRAU, A. (2000). Quelle mort pour demain? Essais d'anthropologie prospective, Paris, L'Harmattan, coll. "Nouvelles études anthropologiques».

BAUDRY, P. (2006). La place des morts. Enjeux et rites, Paris, L'Harmattan, coll. «Nouvelles études anthropologiques".

BERNARD, J. (2009). Croquemort. Une anthropologie des émotions, Paris, Métailié, coll. «Traversées».

BOURGEOIS, E. (2002). L'art de l'embaumement. Une introduction à la thanatopraxie, Austin (Québec), Éditions Berger.

BRONFEN, E. (dir.) (2006). Six Feet Under. Autopsie unseres Umgangs mit Toten I Autopsy of Our Relation to the Dead, Bielefeld et Leipzig, Kerber.

CHARLIER, P. (2006). Médecin des morts. Récits de paléopathologie, Paris, Fayard.

CHENEY, A. (2006). Body Brokers. Inside America's Underground Trade in Human Remains, New York, Broadway Books.

CLAVANDIER, Gaëlle (2009). Sociologie de la mort. Vivre et mourir dans la société contemporaine, Paris, Armand Colin, coll. "U».

CRAIG, E. (2005). Secrets de cadavres, Montréal, Éditions de l'Homme.

CRETTAZ, B. (2010). Cafés mortels: sortir la mort du silence, Genève, Labor et Fides.

DABOUIS, G. (2010). La mort. Journées de la Maison des Sciences de l'homme Ange-Guépin, Paris, L'Harmattan.

DELMOTTE, B. (2010). Esthétique de l'angoisse. Le Memento mori comme thème esthétique, Paris, Presses universitaires de France, coll. "Ligne d'art».

DI FOLCO, P. (dir.) (2010). Dictionnaire de la mort, Paris, Larousse.

DOMINGUEZ LEIVA, A. (2004). Décapitations. Du culte des crânes au cinéma gore, Paris, Presses universitaires de France, coll. «Littératures européennes».

DOMINGUEZ LEIVA, A. et M. DÉTRIE (2005). Le supplice oriental dans la littérature et les arts, Neuillylès-Dijon, Éditions du Murmure.

DOMINGUEZ LEIVA, A. et S. HUBIER (2008). Délicieux supplices. Érotisme et cruauté en Occident, Neuillylès-Dijon, Éditions du Murmure.

EDELMAN, B. (2010). Ni chose, ni personne. Le corps humain en question, Paris, Hermann.

ERNST, G. (dir.) (1988). La mort dans le texte, Lyon, Presses universitaires de Lyon.

ERNST, G. (dir.) (1983). La mort en toutes lettres, Nancy, Presses universitaires de Nancy.

FOUCAULT, M. (2010). Naissance de la clinique. Nouv. éd., Paris, Presses universitaires de France, coll. "Quadrige».

GODEAU, E. (2007). L'esprit de corps. Sexe et mort dans la formation des internes en médecine, Paris, Maison des Sciences de I'homme, coll. «Ethnologie de France».
GOYETTE, D. (2010). Croque-morts et thanatologues, Waterloo (Québec), Éditions Michel Quintin, coll. "Québec insolite».

GUILLAUD, L. (2010). Le retour des morts. Imaginaire, science, verticalité, Pertuis, Éditions Rouge Profond.

GUILLE-ESCURET, G. (2010). Sociologie comparée du cannibalisme. Gibiers et captifs en Afrique, Paris, Presses universitaires de France.

HARRISON, R. (2003). Les morts, Paris, Éditions Le Pommier.

HENNIG, J.-L. (2007). Morgue. Enquête sur le cadavre et ses usages, Paris, Verticales.

KOZLOVSKY-GOLAN, Y. (2008). The Shaping of the Holocaust Visual Image by the Nuremberg Trials, Göttingen, Wallstein Verlag, coll. "Search and research - Lectures and papers $", n^{\circ} 9$.

LALIBERTÉ, L.-A. et D. TREMBLAY (2010). Les cimetières du Québec, Québec, Éditions GID.

LE BRETON, D. (2008). La chair à vif. De la leçon d'anatomie à la greffe d'organes, édition revue et complétée, Paris, Métailié.

LECOMTE, D. (2011). La maison du mort, Paris, Fayard.

MANDRESSI, R. (2003). Le regard de l'anatomiste. Dissections et invention du corps en Occident, Paris, Seuil.

MOLINIÉ, M. (2006). Soigner les morts pour guérir les vivants, Paris, Empêcheurs de Penser en Rond.

MONTROSS, C. (2007). Body of Work. Meditations on Mortality from the Human Anatomy Lab, New York, The Penguin Press.

MOREL CINQ-MARS, J. (2010). Le deuil ensauvagé, Paris, Presses universitaires de France.

PERALDI, F. (2010). La mort. Séminaire 1985-1988, Montréal, Liber.

PIGAILLEM, H. (2008). Petit dico insolite de la mort. Morts célèbres et absurdes, anecdotes et chroniques, Saint-Victor-d'Épine, City éditions.

PORTIER-KALTENBACH, C. (2007). Histoires d'os et autres illustres abattis, Paris, JC Lattès.

RAMSLAM, K.M. (2001). Cemetery Stories: Haunted Graveyards, Embalming Secrets, and the Life of a Corpse after Death, New York, It Books.

ROACH, M. (2005). Macchabées. La vie mystérieuse des cadavres, Paris, Calmann-Lévy.

SELZER, R. (1987). La chair et le couteau. Confessions d'un chirurgien, Paris, Seuil.

SIMARD, J. et BRAULT, F. (2008). Cimetières. Patrimoine pour les vivants, Québec, Éditions GID.

TAPIE, A. (2010). La vanité: mort, que me veux-tu? Paris, La Martinière.

TESSON-MILLET, M.-C. (2010). Foutre la paix aux morts!, Paris, JC Lattès.

THOMAS, L.-V. (2010 [1975]). Anthropologie de la mort, Paris, Payot, coll. «Bibliothèque scientifique Payot».

THOMAS, L.-V. (2003). La mort (5e éd.), Paris, Presses universitaires de France, coll. «Que sais-je?».

URBAIN, J.-D. (2005). L'archipel des morts. Cimetières et mémoire en Occident, Paris, Payot, coll. «Petite bibliothèque Payot».

VON HAGENS, G. et A. WHALLEY (2008). Body Worlds. The Original Exhibition of Real Human Bodies, [s.I.], Arts \& Sciences Verlag.

WALTER J. (2005). La Shoah à l'épreuve de l'image, Paris, Presses universitaires de France.

\section{NOUVELLES PARUTIONS - ÉTUDES SUR LA \\ MORT \\ Serge Gariépy}

ADRIAENSEN, M.-C. et E.C.J. RETSYLAM (2010). La personne âgée face au deuil: comment lui venir en aide, Bruxelles, De Boeck.

AKHTAR, S. (2010). The Wound of Mortality: Fear, Denial, and Acceptance of Death, Lanham (MD), Rowman and Littlefield Publishers.

BENATAR, D. (2010). Life, Death and Meaning: Key Philosophical Readings on the Big Questions, Lanham (MD), Rowman \& Littlefield Publishers.

CARROLL, M. et J. REMPEL (dir.) (2010). Living through the Dead: Burial and Commemoration in the Classical World, Oxford, Oxbow Books Limited.

COQUET, M. (2010). Comprendre la mort pour connaître la vie, Monaco, Alphée.

CRETTAZ, Bernard (2010). Cafés mortels: sortir la mort du silence, Genève, Labor et Fidès.

FOLCO, P. Di (2010). Dictionnaire de la mort, Paris, Larousse.

JACQUES, J. (2010). Les Saisons du deuil, Outremont, Québécor.

JOHNSTON, M. (2010). Surviving Death, Princeton (NJ), Princeton University Press.

KAUFFMAN, J. (dir.) (2010). The Shame of Death, Grief, and Trauma, New York (NY), Routledge.

KELLEHEAR, A. (2010). The Study of Dying: From Autonomy to Transformation, Cambridge, Cambridge University Press.

KOPPELMAN, K.L. (2010). Wrestling with the Angel: Literary Writings and Reflections on Death, Dying and Bereavement, Amityville, Baywood Publishing.

MICHAELSON, J. (2010). Step into Our Lives at the Funeral Home, Amityville, Baywood Publishing.

MONROE, B. (2010). Brief Interventions with Bereaved Children, Oxford, Oxford University Press.

PHILLIPPY, P. (2010). Women, Death and Literature in Post-Reformation England, Cambridge, Cambridge University Press.

SIMON, J.K. (2010). Solution Focused Practice in End-Of-Life and Grief Counseling, New York (NY), Springer.

SMITH, S.E. (2010). To Serve the Living: Funeral Directors and the African American Way of Death, Cambridge, Harvard University Press.

SZABO, J.F. (2010). Death and Dying: An Annotated Bibliography of the Thanatological Literature, Lanham (MD), Scarecrow Press.

WIMPENNY, Peter (2010). Grief, Loss and Bereavement Care: An Evidence Based Approach for Health and Social Care, New York (NY) Routledge. 